\title{
Combinational treatment with microRNA-133b and cetuximab has increased inhibitory effects on the growth and invasion of colorectal cancer cells by regulating EGFR
}

\author{
JIANYU ZHOU ${ }^{1}$, LV LV $^{2}$, CHANGWEI LIN ${ }^{1}$, GUI HU ${ }^{1}$, YIHANG GUO ${ }^{1}$, \\ MEIRONG WU ${ }^{3}$, BUNING TIAN ${ }^{1}$ and XIAORONG LI ${ }^{1}$ \\ Departments of ${ }^{1}$ General Surgery and ${ }^{2}$ Emergency; ${ }^{3}$ Operation Center, \\ The Third Xiangya Hospital of Central South University, Changsha, Hunan 410013, P.R. China
}

Received August 6, 2014; Accepted June 3, 2015

DOI: $10.3892 / \mathrm{mmr} .2015 .4046$

\begin{abstract}
Colorectal cancer (CRC) is the third most common cancer with a very poor prognosis predominantly due to its high rate of tumor invasion and migration, and its resistance to anti-epidermal growth factor receptor (EGFR) therapy. Although CRC has been widely studied, the underlying molecular mechanism remains to be elucidated. MicroRNA (miR)-133b has been demonstrated to act as a tumor suppressor in several human cancer types by regulating EGFR. However, the detailed involvement of miR-133b and EGFR in CRC cells remain to be elucidated. The present study used reverse transcription quantitative polymerase chain reaction and characterized the downregulation of the expression levels of miR-133b in CRC tissues and cell lines. Cell functional assays demonstrated that restored expression of miR-133b inhibited the growth and invasion of CRC cells. In addition, a luciferase reporter assay revealed that miR-133b directly targeted EGFR and repressed its expression levels in CRC cells. Additionally, combination treatment with miR-133b mimics and the monoclonal anti-EGFR antibody, cetuximab, which is approved and frequently used for treating patients with $\mathrm{CRC}$, exhibited improved inhibitory effects on the growth and invasion of CRC cells compared with treatment with either alone. Taken together, the present study characterized the role of the miR-133b/EGFR interaction in CRC cells and this suggested the combinational therapy with cetuximab and miR-133b was positive and may be a potential novel treatment for patients with $\mathrm{CRC}$ in the future.
\end{abstract}

Correspondence to: Dr Xiaorong Li, Department of General Surgery, The Third Xiangya Hospital of Central South University, 138 Tongzipo Road, Changsha, Hunan 410013, P.R. China E-mail: xiaorongli1407@163.com

Key words: miR-133b, epidermal growth factor receptor, colorectal cancer, cetuximab

\section{Introduction}

Colorectal cancer (CRC) is the third most common type of cancer with a very poor prognosis predominantly due to its high rate of tumor invasion and migration, and resistance to anti-EGFR therapy (1). Although CRC has been widely studied, the underlying molecular mechanism remains to be elucidated.

MicroRNAs (miRNAs), a class of small non-coding RNAs, can negatively regulate gene expression by targeting the 3'-untranslated regions (UTRs) of specific target mRNAs to cause either mRNA degradation or translational inhibition (2). miRNAs are important in s all biological activities in mammals (3) and accumulating evidence reveals that miRNAs are also involved in cancer-associated processes, including proliferation, migration and apoptosis (4). Therefore, miRNAs are being used as diagnostic and prognostic biomarkers for certain types of cancer and also as therapeutic targets. Previously, miR-133b was reported to be significantly dysregulated in certain types of tumor, including gastric cancer (5-7), bladder cancer (8), prostate cancer $(9)$ and lung cancer $(10,11)$. In $\mathrm{CRC}$, low expression of miR-133b was reported to be correlated with poor survival and metastasis (12). However, the molecular mechanisms underlying miR-133b in CRC cells remain to be elucidated.

The EGFR signaling pathway regulates cell proliferation, differentiation, migration and apoptosis, and widely participates in tumorigenesis (13-15). Therefore, EGFR has been used as a therapeutic molecular target for several metastatic types of cancer, including CRC. Several agents targeting EGFR, including cetuximab, have been approved and are currently used for treating patients with CRC (16). Although certain advances in CRC therapy have been made, patient survival remains poor. This is predominantly due to the fact that patients with CRC, which are sensitive to cetuximab almost always develop resistance within several months. Previous studies have demonstrated that miRNAs are involved in the EGFR network during cancer oncogenesis (16) and other previous studies revealed miRNAs to be associated with the therapeutic response in several cancer cells. For example, miR-221, miR-222, miR-30 and miR-130a were reported 
to be involved in gefitinib resistance in non-small cell lung cancer cells (17-19). In CRC cells, the cell lines sensitive and resistant to cetuximab expressed different sets of miRNAs following treatment with cetuximab (20). This suggests that certain miRNAs are closely associated with the therapeutic response in CRC and suggests that they may be important for overcoming drug resistance in patients with CRC.

The present study determined the involvement of miR-133b and EGFR in CRC cells. The findings indicated that miR-133b is downregulated in human CRC tissues and cell lines, and increased expression levels of miR-133b inhibited the growth and invasion of CRC cells. In addition, miR-133b directly targeted EGFR and inhibited its expression in CRC cells. Silencing of EGFR using TALEN-based knock-out and treatment with cetuximab inhibited CRC cell growth and invasion in vitro. Additionally, combinational treatment of miR-133b mimics and cetuximab exhibited improved inhibitory effects on the growth and invasion of CRC cells compared with either of treatments alone. Taken together, the present study characterized the role of the miR-133b/EGFR interaction in CRC cells and this suggested the combinational therapy with cetuximab and miR-133b was positive and may be a potential novel treatment for patients with CRC in the future.

\section{Materials and methods}

Tissue samples and cell lines. A total of nine CRC tumor tissue samples and three normal tissue samples were collected from The Third Xiangya Hospital of Central South University (Hunan, China). The present study was approved by the Independent Ethical Committee of Central South University. The samples were stored at $-80^{\circ} \mathrm{C}$ until used. A total of five CRC cell lines, HT-29, SW480, SW620, Caco-2 and HCT-116, (American Type Culture Collection, Rockville, MA, USA) were used. The cells were grown routinely in H-Dulbecco's modified Eagle's medium (DMEM; Gibco Life Technologies, Carlsbad, CA, USA), containing $10 \%$ fetal bovine serum (Gibco Life Technologies) and cultured at $37^{\circ} \mathrm{C}$ in humidified air of $5 \% \mathrm{CO}_{2}$.

Antibody and cetuximab. Rabbit polyclonal anti-EGFR antibody (1:200) was purchased from Abcam (Hong Kong, China) and Cetuximab $\left(100 \mu \mathrm{g} / \mathrm{ml}^{-1} ; 2 \mathrm{mg} / \mathrm{ml}^{-1}\right)$ was purchased from Merck Pharma GmbH (Erbitux, Darmstadt, Germany).

RNA isolation and reverse transcription quantitative polymerase chain reaction $(R T-q P C R)$. The total RNA was extracted from cells and the patient samples using TRIzol reagent (Invitrogen Life Technologies, Carlsbad, CA, USA) and $\sim 500 \mathrm{ng}$ extracted total RNA was reverse-transcribed into cDNA using the Primer Script RT reagent kit (Takara Bio, Inc., Otsu, Japan). The relative mRNA expression levels of EGFR were detected using a SYBR-Green qPCR assay (Takara Bio, Inc.) performed on an ABI Prism 7700 (Applied Biosystems, Foster City, CA, USA). $\beta$-actin was used as a control for normalization. The specific primer sequences used were: EGFR, forward 5'-CTTCACACATACTCCTCCTC-3' and reverse 5'-TCTCCATCACTTATCTCCTT-3'; $\beta$-actin, forward 5'-AGGGGCCGGACTCGTCATACT-3' and reverse 5'-GGCGGCACCACCATGTACCCT-3'. The relative expression levels of miR-133b were determined using an mirVana $^{\mathrm{TM}}$ qRT-PCR miRNA Detection kit (Ambion, Austin, TX, USA), according to the manufacturer's instructions. The specific primers for miRNA-133b and U6 were purchased from GeneCopoeia, Inc. (Rockville, MD, USA). The expression levels of U6 were used as an endogenous control. All experiments were performed in triplicate and the relative expression levels were calculated using the $2^{-\Delta \Delta C t}$ method.

Dual luciferase reporter assay. The 3'UTR of the wildtype EGFR (position 50-56 of EGFR 3'UTR; GGACCAA), and a variant containing mutations (GCAGCTA) in the putative binding site, were inserted downstream of the firefly luciferase reporter into the psiCHECK-2 vector (Promega, Madison, WI, USA). The corresponding mutant construct was created by mutating the seed regions of the miR-133b binding sites and was termed 3'-UTR mut EGFR. The primer sequences used were: 3'UTR EGFR, forward 5'-CCGCTCGAGCCACGGAGGATAGTAT GAG-3' and reverse 5'-GTTGCGGCCGCGGAAGCCTT GAAGCAGAAC-3'; 3'-UTR mut EGFR, forward 5'-GTTGCG GCCGCCCACGGAGGATAGTATGAG-3' and reverse 5'-CCGCTCGAGGGAAGCCTTGAAGCAGAAC-3'. The cells were transfected using lipofectamine 2000 (Invitrogen Life Technologies) and were co-transfected with reporter constructs with pre-miR-133b, anti-miR-133b, pre-scramble or anti-scramble. An untreated group was used as a control. Luciferase activity was detected $48 \mathrm{~h}$ after transfection using a dual-luciferase reporter gene assay kit (Promega), and the luciferase activities in each group were determined using an LD400 luminometer (Promega.). Renilla luciferase activity was normalized to firefly luciferase activity. Flow cytometery was performed to determine cell apoptosis using an Annexin V-fluoresecin isothiocyanate (FITC) Apoptosis Detection kit (BD Biosciences, Frankin Lakes, New Jersey, United State). At $24 \mathrm{~h}$ post-transfection, the cells were harvested and washed twice with cold PBS. Subsequently, $10^{6}$ cells were resuspended in $200 \mu \mathrm{l}$ binding buffer with $10 \mu \mathrm{l}$ Annexin-FITC and 5 $\mu \mathrm{l}$ propidium iodide, followed by incubation in the dark for $30 \mathrm{~min}$. Finally, $300 \mu \mathrm{l}$ binding buffer was added and the cells were assessed using flow cytometric analysis (Moflo XDP; Beckman Coulter, Brea, CA, USA).

Cell proliferation assay. The cells were seeded into 96-well plates at a density of $5 \times 10^{3}$ cells/well and were incubated for 24,48 and $72 \mathrm{~h}$ at $37^{\circ} \mathrm{C}$ with $5 \% \mathrm{CO}_{2}$. The viability of the cells was assessed using an MTT assay. Briefly, MTT $(10 \mathrm{mg} / \mathrm{ml})$ was added to the cells and incubated for $3 \mathrm{~h}$ at $37^{\circ} \mathrm{C}$ The reaction was terminated by removal of the supernatant, followed by the addition of $200 \mu \mathrm{l}$ dimethyl sulfoxide and gently pipetting. Following a $2 \mathrm{~h}$ incubation, the optical density of each well at $570 \mathrm{~nm}$ was measured with a BioRad microplate reader (Bio-Rad, Hercules, CA, USA). Each assay was performed in triplicate.

Cell cycle analysis by flow cytometric analysis. The cells were digested, washed with phosphate-buffered saline (PBS), and subsequently fixed in $70 \%$ ethanol at $4^{\circ} \mathrm{C}$ overnight. The fixed cells $\left(1 \times 10^{6}\right)$ were washed with PBS and resuspended in staining solution, containing $50 \mu \mathrm{g} / \mathrm{ml}$ propidium iodide, 
A

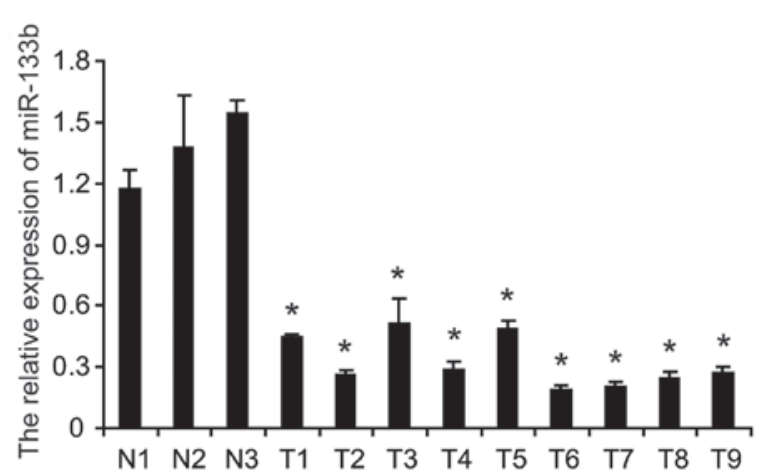

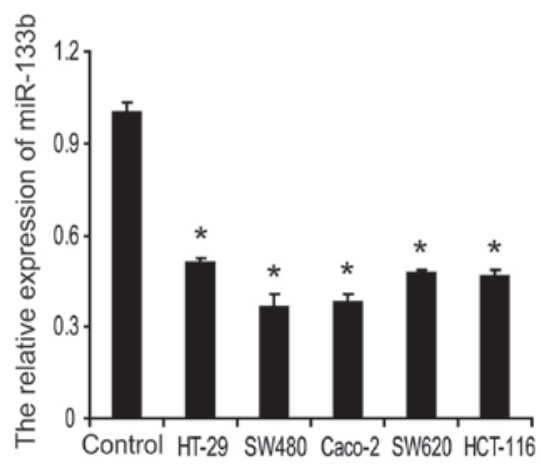

Figure 1. Expression levels of miR-133b decreases in CRC tumor types and cell lines. (A) The expression of miR-133b was decreased in nine CRC tumor samples (T1-T9) compared with that of three normal tissue samples (N1- N3), as detected by RT-qPCR. (B) Decreased expression levels of miR-133b were detected in CRC HT-29, SW480, Caco-2, SW620 and HCT-116 cell lines compared with the expression level in normal colonic mucosa epithelial cells isolated and pooled from three adjacent non-cancerous tissues (Control), as detected by RT-qPCR. The data are expressed as the mean \pm standard deviation ( ${ }^{*} \mathrm{P}<0.05$, compared with normal tissue or normal cells). CRC, colorectal cancer; RT-qPCR, reverse transcription quantitative polymerase chain reaction; miR, microRNA.

$1 \mathrm{mg} / \mathrm{ml}$ RNase A and $0.1 \%$ Triton X-100 in PBS. Following incubation for $30 \mathrm{~min}$ at $4^{\circ} \mathrm{C}$, the stained cells were analyzed on a flow cytometer (Beckman Coulter).

Cell invasion assay. Cell invasion was determined using a transwell assay. Briefly, the cells were suspended in serum-free medium and aliquots $\left(1 \times 10^{5}\right.$ cells $)$ of the prepared cell suspension were added into the upper chamber. The lower chamber was filled with $1 \mathrm{ml}$ DMEM, containing fetal bovine serum $(10 \%)$. Following incubation for $24 \mathrm{~h}$ at $37^{\circ} \mathrm{C}$, the cells remaining on the upper side of the membrane were removed using a cotton swab, while the cells, which had migrated through the membrane were fixed with $75 \%$ alcohol and stained with $1 \%$ crystal violet for $20 \mathrm{~min}$. The invasive cells were subsequently counted and images were captured using an inverted microscope (Nikon, Tokyo, Japan).

Transcription activator-like effector nucleases (TALEN)mediated knockout of the EGFR gene in Caco-2 cells. The present study used TALEN technology to knock out the EGFR gene in Caco-2 cells. TALENs designed to target the EGFR gene were purchased from Sidansai Biotechnology (Shanghai, China). The cells in 24-well plates were transfected with $400 \mathrm{ng}$ TALEN expression plasmids (EGFR-TALEN) or negative control (TALEN-NC) using Lipofectamine 2000 (Invitrogen Life Technologies), according to the manufacturer's instructions. Western blotting was performed to determine the protein expression levels of EGFR and confirm the efficiency of the TALEN mediated knockout.

\section{Results}

miR-133b is decreased in CRC tumor samples and cell lines. To determine the potential clinical and pathological implications of an altered expression of miR-133b in CRC tumor samples, the present study investigated the expression levels of miR-133b in nine CRC tumor samples and three normal tissue samples using RT-qPCR. As shown in Fig. 1A, the expression levels of miR-133b were significantly lower in CRC tumor samples compared with the normal tissue samples. Furthermore, the abundance of miR-133b in five
CRC cell lines was demonstrated. Compared with normal colonic mucosa epithelial cells isolated and pooled from three adjacent non-cancerous tissue samples, the expression levels of miR-133b in the five CRC cell lines were significantly reduced to different extents, markedly lower in the SW480 and Caco-2 cells (Fig. 1B). These results suggested that the expression levels of miR-133b were significantly decreased in human CRC specimens and cell lines, which suggested that it may be involved in the pathogenesis of CRC.

miR-133b targets and represses the expression of EGFR in CRC cells in vitro. To determine whether miR-133b directly targets EGFR in CRC cells, a luciferase assay was performed. A region of $501 \mathrm{bp}$ length of the wild-type 3'-UTR of EGFR, containing the predicted miR-133b target sites, was amplified and inserted into the resulting amplicon downstream of a luciferase reporter gene (termed wt-EGFR). A mutant version of the 3'-UTR of EGFR lacking the miR-133b binding sites (named mut-EGFR), was also constructed. The SW480 and Caco-2 cells were co-transfected with wt-EGFR or mut-EGFR vectors and either the miR-133b mimics (pre-miR-133b), miR-133b inhibitor (anti-miR-133b) or their respective scrambled controls (pre-scramble and anti-scramble). The results showed that miR-133b significantly suppressed the luciferase activity of the reporter gene, containing the 3'UTR of EGFR compared with the controls, however, this was significantly rescued when the miR-133b binding sites were absent in the SW480 and Caco-2 cells (Fig. 2A and B). Furthermore, the inhibitory effect of miR-133b on EGFR expression was assessed. RT-qPCR analysis demonstrated that an sincreased expression of miR-133b significantly decreased the mRNA expression levels of EGFR compared with the cells transfected with the control (Fig. 2C and D). Taken together, these results confirmed that miR-133b directly targeted EGFR and repressed its expression levels in CRC cells.

miR-133b inhibits the growth and invasion of CRC cells. To investigate the roles of miR-133b in the CRC cells, the expression of miR-133b was restored in SW480 and Caco-2 cells. This resulted in a lower expression of miR-133b in the selected 
A

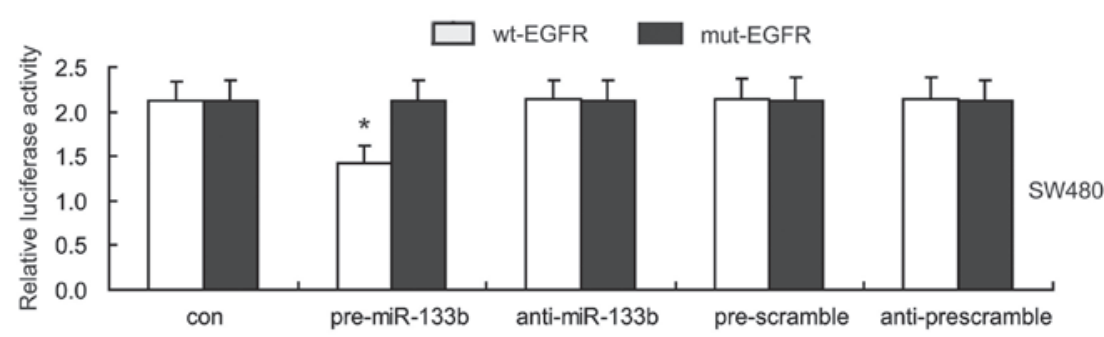

B

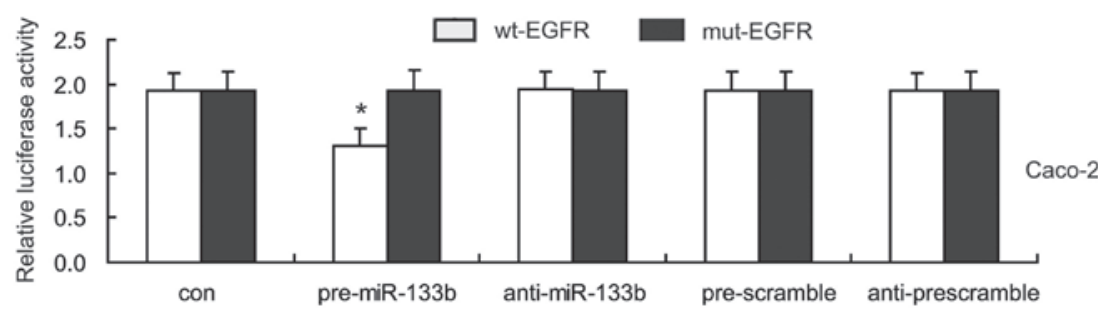

C
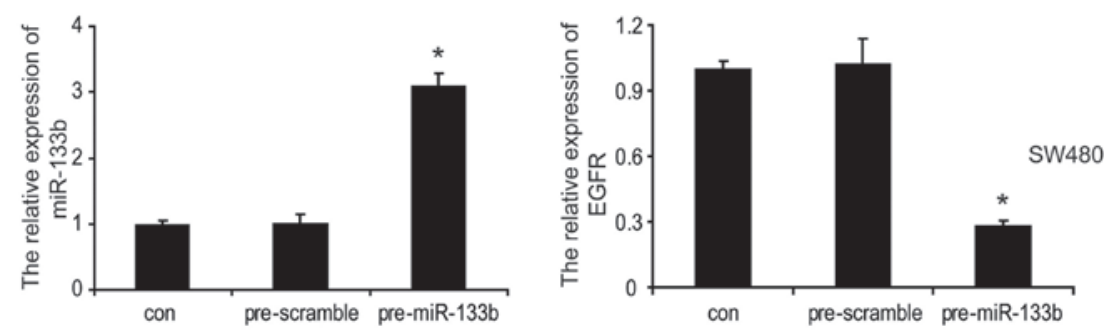

D
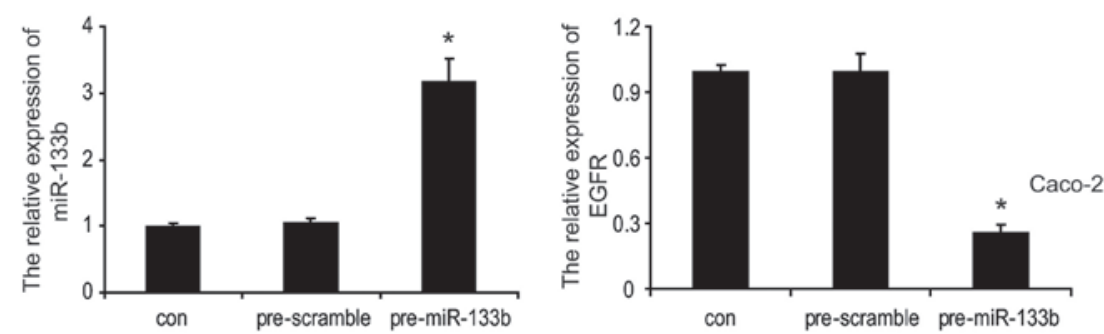

Figure 2. miR-133b directly targets and represses the expression levels of EGFR. (A and B) The repression of luciferase activity by the 3'UTR of EGFR was dependent on miR-133b and the mutated 3'UTR of EGFR abrogated the miR-133b mediated repression of luciferase activity in SW480 and Caco-2 cells, respectively. (C and D) Reverse transcription quantitative polymerase chain reaction was performed to detect the expression levels of miR-133b and EGFR following transfecting with pre-miR-133b, pre-scramble or control in SW480 and Caco-2 cells, respectively. The data are expressed as the mean \pm standard deviation ( $\mathrm{P}<0.05$, compared with the control). UTR, untranslated region; con, control; wt. wild-type; mut, mutant; miR, microRNA; EGFR, epidermal growth factor receptor.

CRC cell lines, by liposomal delivery. The effect of miR-133b on cell growth and invasion was investigated. An MTT assay demonstrated that the overexpression of miR-133b decreased the cell viability in SW480 and Caco-2 cells (Fig. 3A and B). It was also revealed that miR-133b marginally increased the percentage of G1 phase cells in SW480 and Caco-2 cells (Fig. 3C and D), as determined by flow cytometric analysis. A Transwell assay indicated the forced expression of miR-133b significantly inhibited the cell invasion ability compared with the control group in SW480 and Caco-2 cells (Fig. 3E and F). These results suggested that miR-133b, which serves as a tumor suppressor, is important in the control of growth and invasion of CRC cells in vitro.

Silencing of EGFR by TALEN inhibits the growth and invasion of Caco-2 cells. Based on the above finding that miR-133b inhibited the cell growth and invasion, at least in part, by downregulating the expression levels of EGFR, the present study hypothesized that inhibiting the expression of
EGFR by other approaches would also have similar inhibitory effects. EGFR was knocked out using TALEN technology, a highly effective approach for the targeted knockout of genes in mammalian cells. Western blotting was performed to confirm the TALEN-mediated knock-out efficiency in Caco-2 cells. As shown in Fig. 4A, the EGFR gene was silenced effectively in the EGFR-TALEN vector transfected cells. As expected, silencing of EGFR by TALEN decreased the cell viability (Fig. 4B), increased the percentage of cells in the G1 phase (Fig. 4C) and inhibited the invasiveness (Fig. 4D) of Caco-2 cells. This suggested that EGFR silencing may be a common effective strategy to inhibit the growth and invasion in CRC cells.

Combination of miR-133b and cetuximab increases the inhibitory effects on the growth and invasion of CRC cells. Cetuximab is a chimeric monoclonal antibody, which binds to EGFR and competitively inhibits ligand binding to suppress tumor proliferation. Cetuximab has been approved and is used 
A

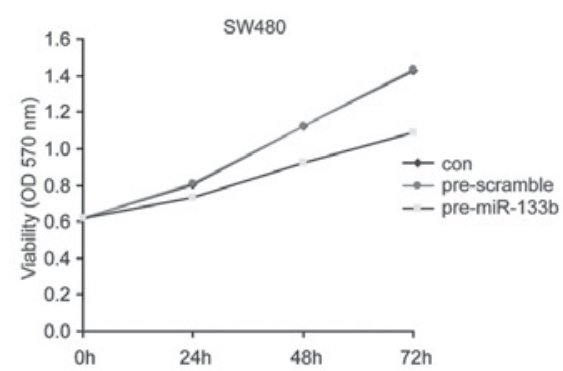

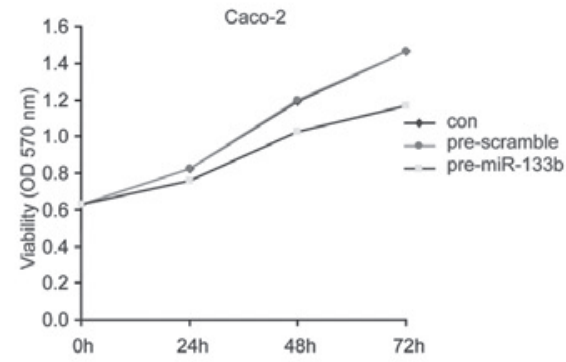

C
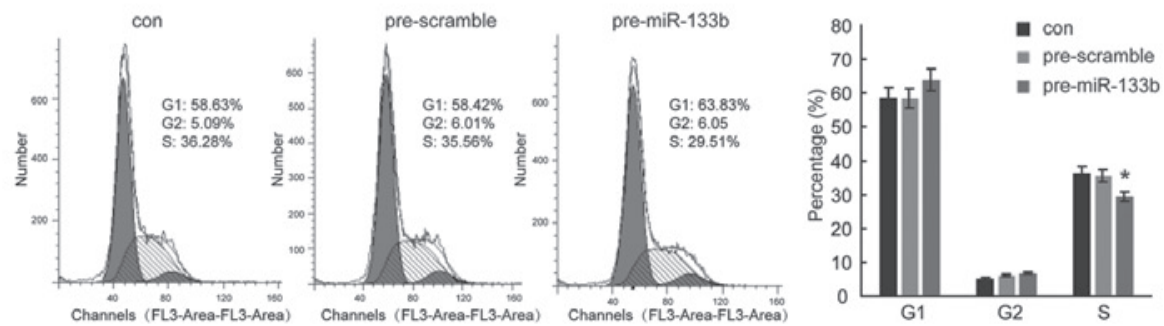

SW480

D
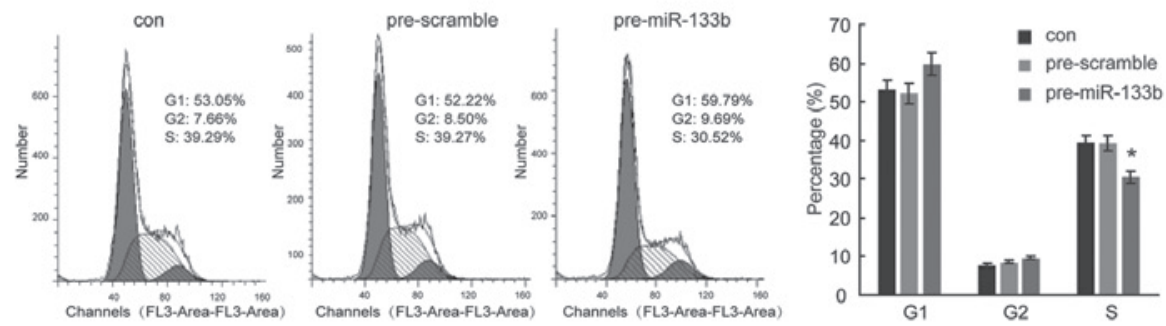

$\mathbf{E}$

con

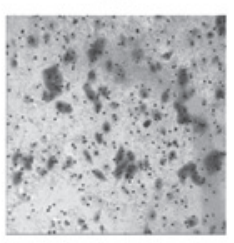

con

$\mathbf{F}$

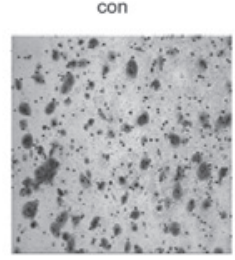

pre-scramble

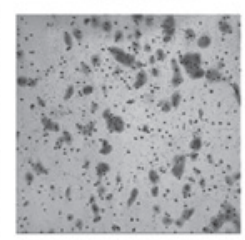

pre-scramble

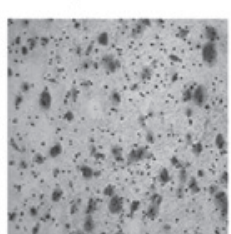

pre-miR-133b

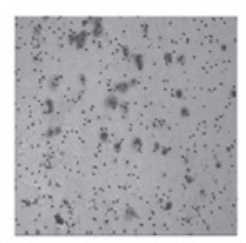

pre-miR-133b

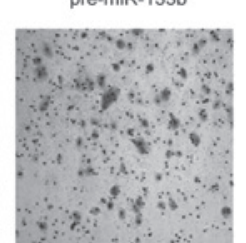

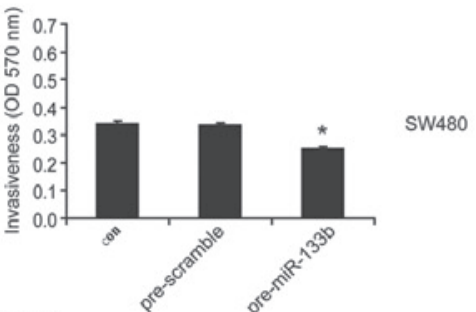

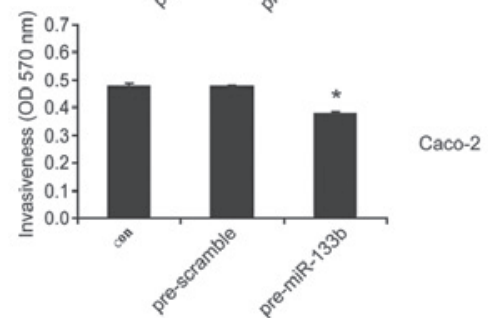

Figure 3. miR-133b inhibits the growth and invasion of colorectal cancer cells. Following transfection with pre-miR-133b, pre-scramble or control in SW480 and Caco-2 cells, tha (A and B) cell viability, $(\mathrm{C}$ and $\mathrm{D}$ ) the percentage of cells in the G1, G2 and S phases and (E and F) the cell invasiveness were determined using MTT, flow cytometry and transwell assays, respectively (magnification, x100). The data are presented as the mean \pm standard deviation ("P $<0.05$, compared with the control). miR, microRNA; con, control; OD, optical density.

for the treatment of patients with CRC. The present study aimed to determine whether the combination of miR-133b and cetuximab provided an improved antitumor effect in CRC cells. To carry this out, the SW480 and Caco-2 cells were treated with cetuximab following transfection with the miR-133b mimics or scramble control. Notably, the combination treatment of miR-133b and cetuximab exhibited an increased inhibitory effect on the cell viability (Fig. 5A and B) and invasion (Fig. 5E and F) of the SW480 and Caco-2 cells compared with treatment with either miR-133b or cetuximab alone. It was also demonstrated that combinational treatment had a marginally increased inhibitory effect on cell cycle progression into $\mathrm{S}$ phase, as determined by flow cytometric analysis (Fig. 5C and D). These data suggested that this was a promising option of a combinational therapy for patients with CRC.

\section{Discussion}

The present study demonstrated that miR-133b is downregulated in CRC tissue samples and cell lines compared with normal tissue samples and cells. When the expression levels 
A

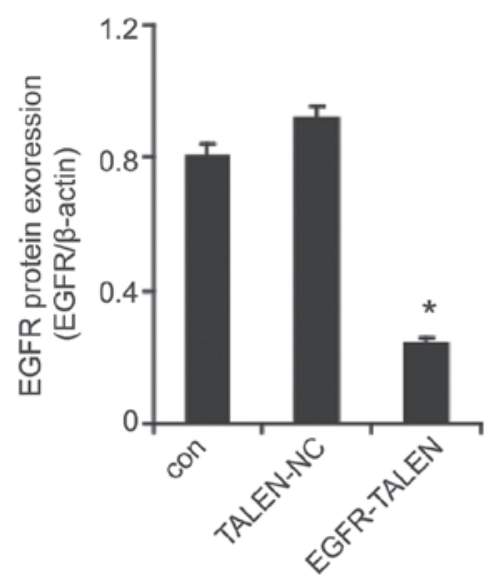

B

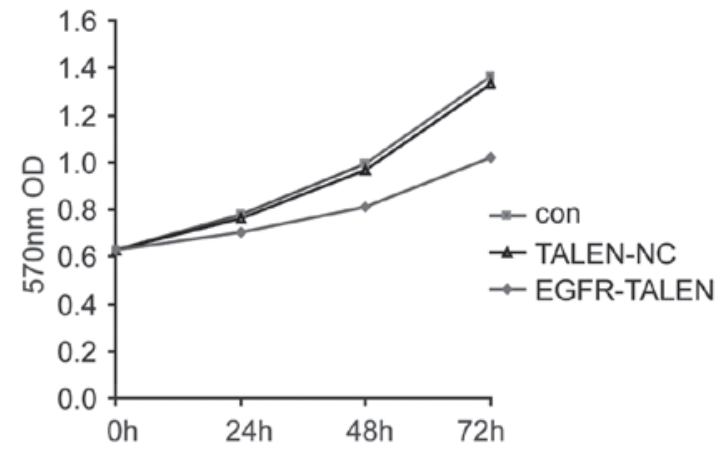

C
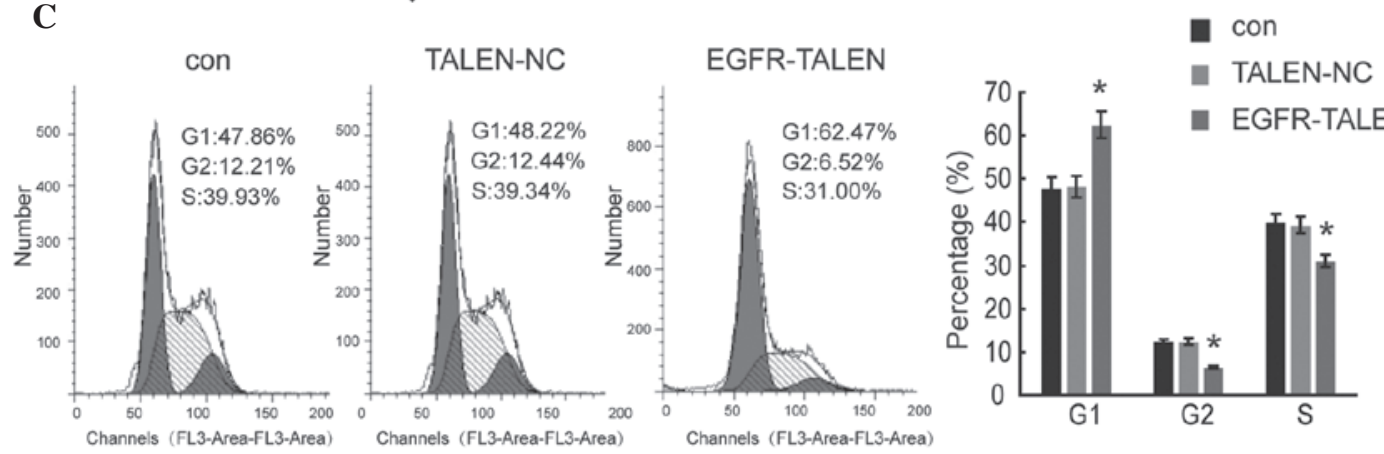

D

con

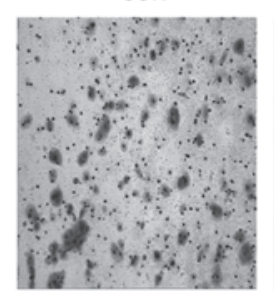

TALEN-NC

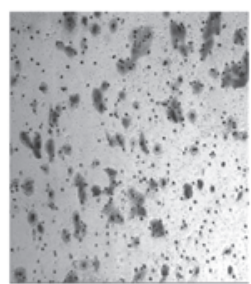

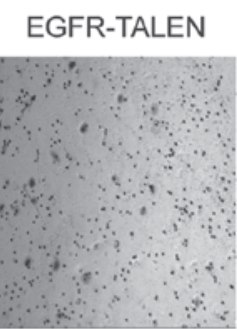

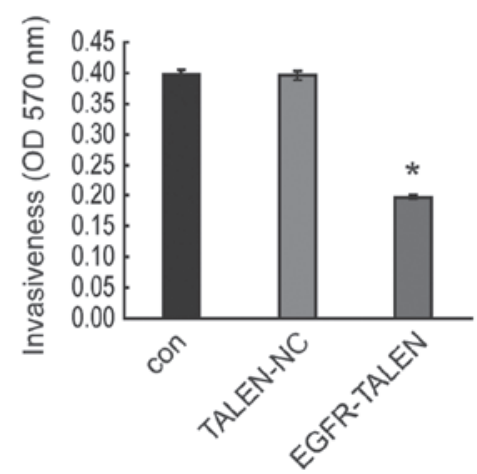

Figure 4. Silencing of EGFR by TALEN inhibits the growth and invasion of Caco-2 cells. (A) Following knock-out using TALEN technology in Caco-2 cells, the protein expression levels of EGFR were determined by western blotting to confirm the TALEN-mediated knock-out efficiency. (B) The cell viability, (C) the percentage of cells in the G1, G2 and S phases and (D) the cell invasiveness were determined by MTT assay, flow cytometric analysis and transwell assay, respectively (magnification, $\mathrm{x} 100$ ). The data are shown as the mean \pm standard deviation (" $\mathrm{P}<0.05$, compared with the control). TALEN, transcription activator-like effector nucleases; con, control; OD, optical density; NC, negative control; EGFR, epidermal growth factor receptor.

of miR-133b were restored, the growth and invasion of CRC cells was inhibited, suggesting that miR-133b may function as a tumor suppressor in the development of CRC. It was also revealed that EGFR is a direct functional target of miR-133b in CRC cells and restored expression of miR-133b repressed the expression of EGFR. Therefore, the present study speculated that low expression levels of miR-133b contributed, at least in part, to EGFR-mediated cell growth and migration in CRC cells. The loss of EGFR by TALEN-based knockout had similar inhibitory effects on the growth and invasion of Caco-2 cells in vitro, which suggested that EGFR silencing may be a common effective strategy to inhibit the growth and invasion in CRC cells. Additionally, combinational treatment with miR-133b mimics and the EGFR monoclonal antibody, cetuximab, demonstrated an increased inhibitory effect on the cell growth and invasion of SW480 and Caco-2 cells compared with either miR-133b or cetuximab alone. These data suggested that this combinational therapy may be a promising choice for treating patients with $\mathrm{CRC}$ in the future. miRNA alterations are involved in the initiation and progression of human cancer. miRNA acts as a tumor suppressor or promoter depending on its target genes (21). Therefore, the miRNA and its targets can shed light on the molecular mechanism underlying cancer progression and provide useful potential therapeutic targets for the clinical treatment of certain types of cancer. miR-133b, a muscle-specific miRNA (22), has previously been reported to be involved in other types of tumor (4-9). The expression of miR-133b is frequently decreased and acts as a tumor suppressor in gastric cancer $(5,6)$, osteosarcoma (23) and esophageal squamous cell carcinoma (24), by negatively regulating the expression of FGFR1, MET and FSCN1, 
A

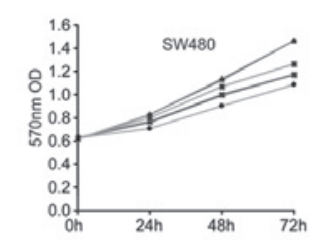

C

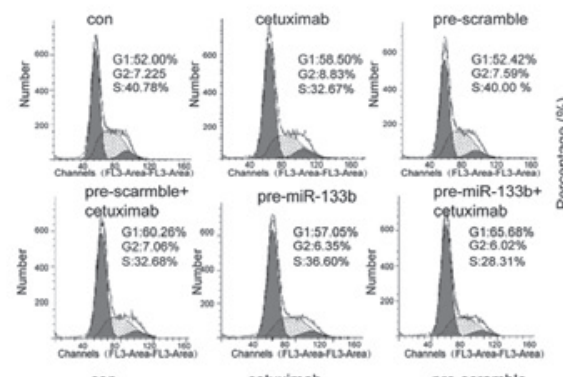

D

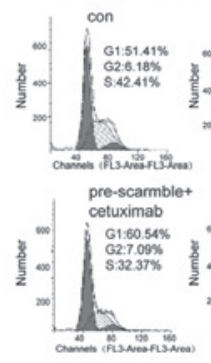

con

E

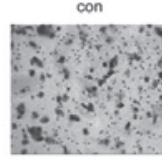

pre-scarmble+
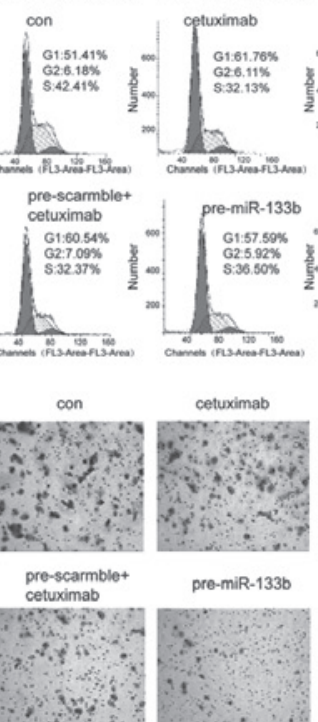

cetuximab

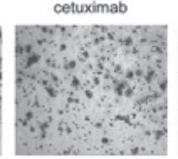

pre-miR-133b
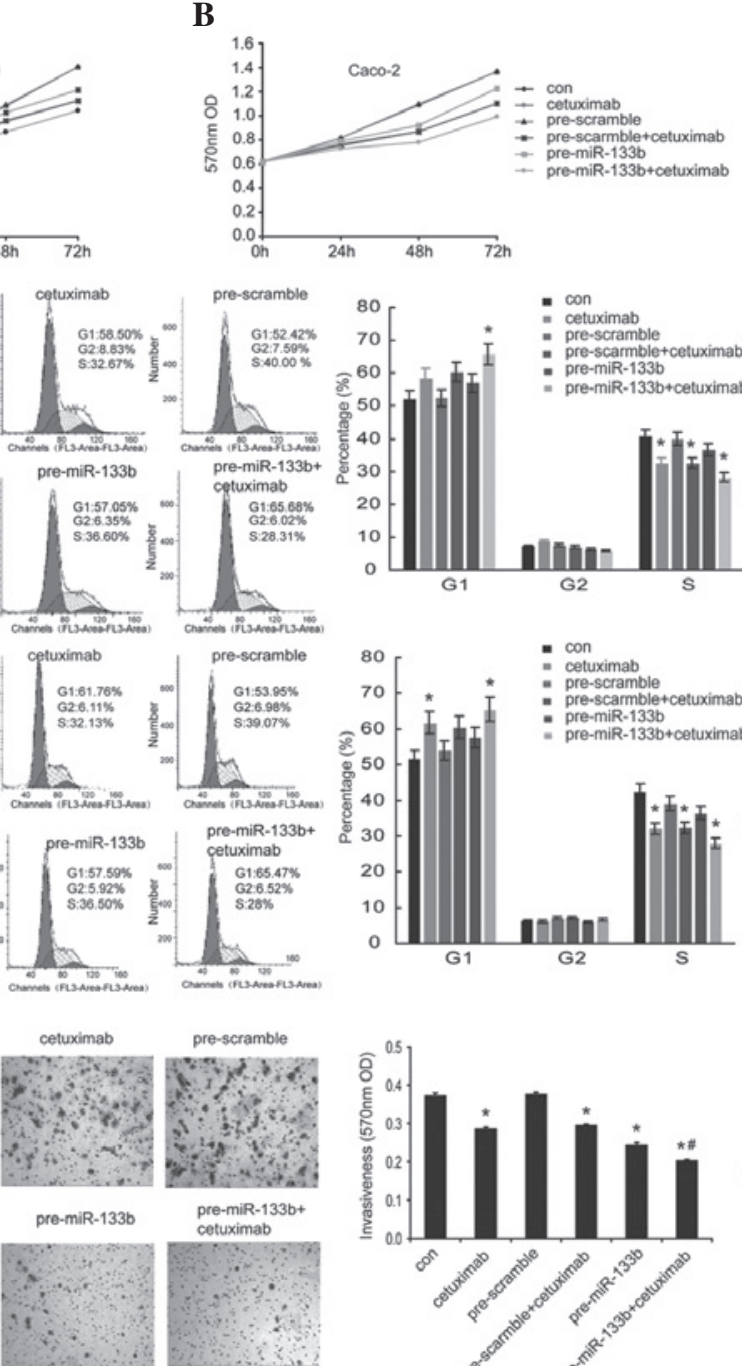

B
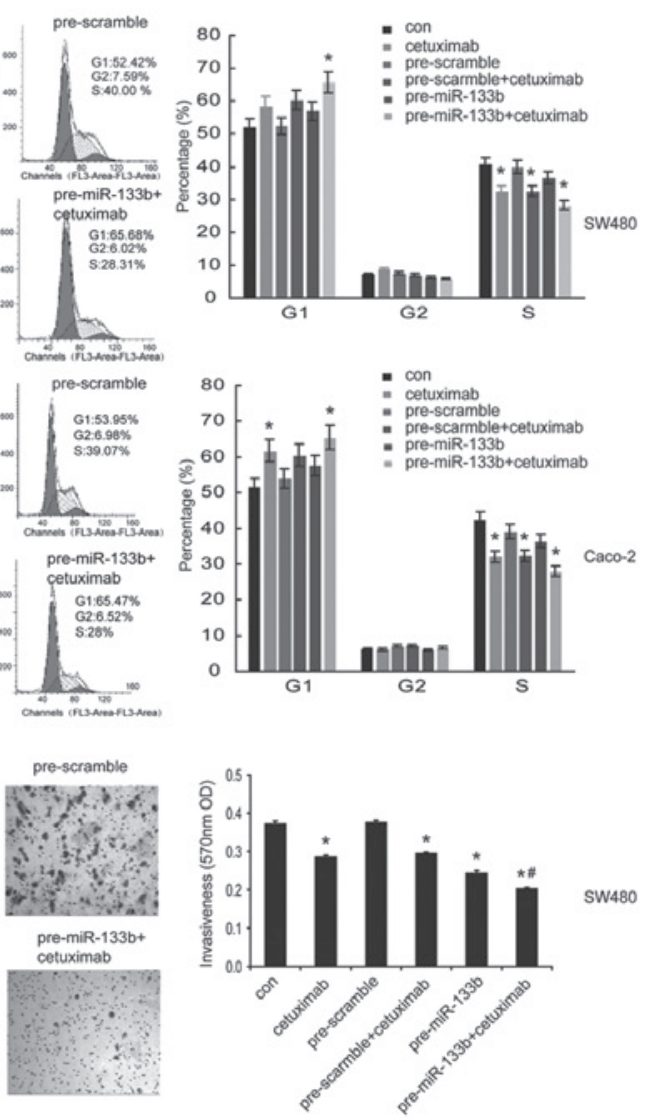

$\mathbf{F}$
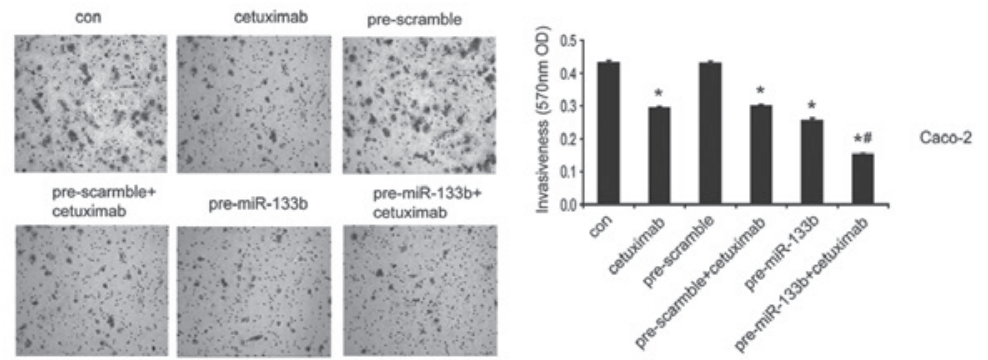

Figure 5. Combination of miR-133b and cetuximab increases the inhibitory effects on the growth and invasion of colorectal cancer cells. SW480 and Caco-2 cells were transfected with pre-miR-133b or pre-scramble, followed by treatment with cetuximab (10 $\mu \mathrm{M})$ for $24 \mathrm{~h}$. An untreated group were used as a control. (A and B) The cell viability, (C and D) the percentage of cells in the G1, G2 and S phases and (E and F) the cell invasiveness were determined by MTT, flow cytometric analysis and transwell assay, respectively (magnification, $\mathrm{x} 100$ ). The data are shown as the mean \pm standard deviation ( $\mathrm{P}<0.05$, compared with the control; ${ }^{\prime} \mathrm{P}<0.05$, compared with the cetuximab treated groups or the pre-miR-133b transfected groups). miR, microRNA; con, control; OD, optical density.

respectively. However, miRNA-133b is a key promoter of the development of cervical carcinoma through the activation of the ERK and AKT1 pathways (25). The present study confirmed that miR-133b inhibited the cell growth and invasion in CRC cells by targeting EGFR, consistent with previous findings that miR-133b inhibits cell growth and invasion in prostate cancer (8), bladder cancer (7) and non-small-cell lung cancer (9) by downregulating EGFR. Taken together, the present study suggested that miR-133b may broadly inhibit EGFR-induced tumorigenesis.
Cetuximab is an EGFR monoclonal antibody and is currently used in the therapy of patients with metastatic CRC. However, several patients treated with cetuximab fail to respond and those who do initially respond often acquire resistance. This may be a result of the extensive involvement of miRNAs in the regulation of the EGFR signaling pathway and certain miRNAs, including miR-133b, close involvement in therapeutic response of CRC cells treated with cetuximab. CRC SW480 and Caco-2 cells were treated with cetuximab and miR-133b mimics to investigate the efficiency of the 
combinational therapy. Notably, the growth and invasion of SW480 cells sensitive to cetuximab and Caco-2 cells resistant to cetuximab, were effectively inhibited by the combinational treatment compared with either of the treatments alone. Taken together, these findings suggested that anti-EGFR therapy with cetuximab in combination with miR-133b or other miRNAs, which inhibit EGFR, may provide therapeutic benefit for patients with metastatic CRC and other EGFR driven types of tumor.

In conclusion, results of the present study confirmed the miR-133b/EGFR association in CRC cells, in which the loss of miR-133b may result in the increased expression levels of EGFR. This increased expression may endow CRC cells with the ability of improved growth and invasion capacity. The restoration of miR-133b and combinational treatment with cetuximab may be a promising therapeutic strategy for the treatment of metastatic CRC in the future.

\section{Acknowledgements}

This study was supported by the Key Program for International Cooperation Projects of Hunan Province (no. 2011WK2011) and the Natural Science Foundation of China (no. 81172298).

\section{References}

1. Asbagh LA, Vazquez I, Vecchione L, et al: The tyrosine phosphatase PTPRO sensitizes colon cancer cells to anti-EGFR therapy through activation of SRC-mediated EGFR signaling. Oncotarget 5: 10070-10083, =2014

2. Lewis BP, Burge CB and Bartel DP: Conserved seed pairing, often flanked by adenosines, indicates that thousands of human genes are microRNA targets. Cell 120: 15-20, 2005.

3. Kato M and Slack FJ: microRNAs: small molecules with big roles-C. elegans to human cancer. Biol Cell 100: 71-81, 2008.

4. Jansson MD and Lund AH: MicroRNA and cancer. Mol Oncol 6: 590-610, 2012.

5. Qiu T, Zhou X, Wang J, et al: MiR-145, miR-133a and miR-133b inhibit proliferation, migration, invasion and cell cycle progression via targeting transcription factor $\mathrm{Sp} 1$ in gastric cancer. FEBS Lett 588: 1168-1177, 2014.

6. Wen D, Li S, Ji F, et al: miR-133b acts as a tumor suppressor and negatively regulates FGFR1 in gastric cancer. Tumour Biol 34 793-803, 2013

7. Zhao Y, Huang J, Zhang L, et al: MiR-133b is frequently decreased in gastric cancer and its overexpression reduces the metastatic potential of gastric cancer cells. BMC Cancer 14: 34, 2014.

8. Zhou Y, Wu D, Tao J, Qu P, Zhou Z and Hou J: MicroRNA-133 inhibits cell proliferation, migration and invasion by targeting epidermal growth factor receptor and its downstream effector proteins in bladder cancer. Scand J Urol 47: 423-432, 2013.
9. Tao J, Wu D, Xu B, et al: microRNA-133 inhibits cell proliferation, migration and invasion in prostate cancer cells by targeting the epidermal growth factor receptor. Oncol Rep 27: 1967-1975, 2012.

10. Liu L, Shao X, Gao W, et al: MicroRNA-133b inhibits the growth of non-small-cell lung cancer by targeting the epidermal growth factor receptor. FEBS J 279: 3800-3812, 2012.

11. Wu J, Yang T, Li X, et al: Alteration of serum miR-206 and $\mathrm{miR}-133 \mathrm{~b}$ is associated with lung carcinogenesis induced by 4-(methylnitrosamino)-1-(3-pyridyl)-1-butanone. Toxicol Appl Pharmacol 267: 238-246, 2013.

12. Akcakaya P, Ekelund S, Kolosenko I, et al: miR-185 and miR-133b deregulation is associated with overall survival and metastasis in colorectal cancer. Int J Oncol 39: 311-318, 2011.

13. Davis NM, Sokolosky M, Stadelman K, et al: Deregulation of the EGFR/PI3K/PTEN/Akt/mTORC1 pathway in breast cancer: possibilities for therapeutic intervention. Oncotarget 5: 4603-4650, 2014

14. Centuori SM and Martinez JD: Differential regulation of EGFR-MAPK signaling by deoxycholic acid (DCA) and ursodeoxycholic acid (UDCA) in colon cancer. Dig Dis Sci 59: 2367-2380, 2014

15. Wang F, Xiao W, Sun J, Han D and Zhu Y: MiRNA-181c inhibits EGFR-signaling-dependent MMP9 activation via suppressing Akt phosphorylation in glioblastoma. Tumour Biol 35: 86538658,2014

16. Mlcochova J, Faltejskova P, Nemecek R, Svoboda M and Slaby O: MicroRNAs targeting EGFR signalling pathway in colorectal cancer. J Cancer Res Clin Oncol 139: 1615-1624, 2013.

17. Garofalo M, Romano G, Di Leva G, et al: EGFR and MET receptor tyrosine kinase-altered microRNA expression induces tumorigenesis and gefitinib resistance in lung cancers. Nat Med 18: 74-82, 2011.

18. Gu YF, Zhang H, Su D, et al: miR-30b and miR-30c expression predicted response to tyrosine kinase inhibitors as first line treatment in non-small cell lung cancer. Chin Med J (Engl) 126: 4435-4439, 2013.

19. Zhou YM, Liu J and Sun W: MiR-130a overcomes gefitinib resistance by targeting met in non-small cell lung cancer cell lines. Asian Pac J Cancer Prev 15: 1391-1396, 2014.

20. Ragusa M, Majorana A, Statello L, et al: Specific alterations of microRNA transcriptome and global network structure in colorectal carcinoma after cetuximab treatment. Mol Cancer Ther 9: 3396-3409, 2010.

21. Calin GA and Croce CM: MicroRNA signatures in human cancers. Nat Rev Cancer 6: 857-866, 2006.

22. Nielsen S, Scheele C, Yfanti C, et al: Muscle specific microRNAs are regulated by endurance exercise in human skeletal muscle. J Physiol 588: 4029-4037, 2010.

23. Novello C, Pazzaglia L, Cingolani C, et al: miRNA expression profile in human osteosarcoma: role of miR-1 and miR-133b in proliferation and cell cycle control. Int J Oncol 42: 667-675, 2013.

24. Kano M, Seki N, Kikkawa N, et al: miR-145, miR-133a and miR-133b: Tumor-suppressive miRNAs target FSCN1 in esophageal squamous cell carcinoma. Int J Cancer 127: 2804-2814, 2010.

25. Qin W, Dong P, Ma C, et al: MicroRNA-133b is a key promoter of cervical carcinoma development through the activation of the ERK and AKT1 pathways. Oncogene 31: 4067-4075, 2012. 